\title{
CONSTITUCIÓN Y MARCO LEGAL DE UN EXCHANGE EN ARGENTINA ${ }^{12}$
}

\author{
CONSTITUTION \& LEGAL FRAMEWORK \\ OF AN ARGENTINLAN EXCHANGE
}

Rocio Belén Varas Nieva(*)

\begin{abstract}
RESUMEN: La cuarta revolución del nuevo mundo exige información respecto de nuevas tecnologías, análisis de conceptos como blockchain, economía digital y específicamente Exchange. En este trabajo, se estudiará ventajas y desventajas para su creación en torno a su marco legal e impositivo en Argentina, con el objeto de un aumento de la demanda y oferta de criptomonedas por parte de la población.
\end{abstract}

PALABRAS CLAVES: Blockchain - Exchange - Marco Legal - Criptomonedas

ABSTRACT: The fourth revolution of the new world requires information regarding new technologies, analysis of concepts such as blockchain, digital economy, and specifically Exchanges. In this work, the advantages and disadvantages of its creation around its legal and tax framework in Argentina will be studied to increase the demand and supply of cryptocurrencies by the population.

KEYWORDS: Blockchain - Exchange - Legal Framework - Cryptocurrencies

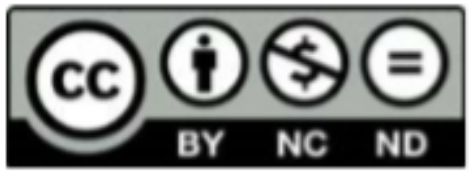

Artículo publicado bajo Licencia Creative Commons Atribución-No Comercial-Sin Derivar. (C) Universidad Católica de Córdoba DOI http://doi.org/10.22529/rbia.2021()12

\footnotetext{
${ }^{1}$ Artículo recibido el 9 de abril de 2021 y aprobado para su publicación el 25 de junio de 2021.

2 El presente es trabajo presentado se realizó en el marco de la DIPLOMATURA EN TECNOLOGÍA BLOCKCHAIN APLICADA A LOS NEGOCIOS Y LAS RELACIONES JURIDICAS, en el año 2020.

(*) Contadora Publica Nacional (UNSE). Diplomada en Tecnología Blockchain aplicada a los negocios y las relaciones jurídicas (UCC). Martillero, Corredor Publico y Corredor Inmobiliario (UES21). Mail: rovaras9@gmail.com
} 


\section{INTRODUCCIÓN}

El presente Trabajo Final, que forma parte de una investigación en curso, consiste en dar claridad a lo establecido en el marco legal para la creación de un Exchange radicado en Argentina con una variedad de servicios digitales que necesitarán de tecnología blockchain para su funcionamiento.

El proyecto apunta a dar claridad a cualquier inversor de poder armar un negocio en una economía digital, en donde las operaciones con criptoactivos tienen un alto rendimiento. Es necesario analizar las ventajas y desventajas de la constitución de un Exchange debido a las dificultades impositivas y legales que el gobierno argentino impone.

A través de este trabajo, se dará claridad para que las personas inviertan sin miedo, eliminando tabúes respecto del marco normativo y sobre todo impositivo de este tipo de negocios, buscando las mejores maneras de reducir el costo para el inversor.

Una de las principales causas por las que se decide invertir en la creación de un Exchange, se debe al contexto de una fuerte crisis de endeudamiento, una macroeconomía con desbalances y una situación social que se manifiesta con fuerza ante los problemas económicos del país. Esto genera la posibilidad, de que el ciudadano invierta en criptomonedas como consecuencia de la existencia de barreras para el ahorro en moneda extranjera, y en moneda argentina debido a la inflación continua.

\section{ANÁLISIS DEL CONTEXTO Y JUSTIFICACIÓN DEL PROYECTO}

\section{Estudio del sector}

El nuevo paradigma digital y la revolución 4.0 de las tecnologías disruptivas intentan demostrar nuevas formas de generar relaciones entre individuos, y en este caso en particular una nueva forma de negocio acompañado de conceptos como blockchain, cash digital, Smart Contracts, economía de opción, etc. Es por eso que se investigará respecto de un caso en particular de un Exchange en Argentina, llamado de ahora en más Cripto del Estero, con el objetivo de prestar los siguientes servicios: 
- Operaciones de intermediación entre un comprador y un vendedor de criptomonedas y stablecoins tal como Bitcoin (BTC), Ether (ETH), Tron (TRX), Theter (USDT), Bitcoin Cash (BCH), Paxos Standard (PAX).

- Venta y compra de criptomonedas y stablecoins ya mencionadas.

- Préstamos de criptomonedas en casas de intercambio recibiendo como contraprestación criptomoneda o moneda Fiat en forma de interés.

En el presente trabajo final, se tomará como base de análisis e investigación las siguientes preguntas:

- ¿Qué es un Exchange? ¿Qué servicios presta?

- ¿Convenía trabajar solo con criptomonedas? ¿Qué beneficios aporta incluir stablecoin en Cripto del Estero?

- $\quad$ En Argentina, ¿están regulados? ¿por quién?

- $\quad \mathrm{Al}$ ser intermediario de una operación, ¿cómo debe actuar el Exchange en el marco impositivo?

- $\quad$ Si soy Exchange, ¿debo ser una sociedad según lo que establece el Código Civil y Comercial de la Nación?

- ¿Qué respaldo jurídico tengo ante una operación entre dos usuarios del Exchange que intercambian criptomonedas?

- ¿ ¿Cuál es la naturaleza jurídica de un Exchange?

Cripto del Estero busca ser una plataforma para el comercio de activos digitales, con un diseño atractivo, confiable y fácil de usar por los usuarios. En este exchange se podrá ver los precios del mercado, realizar depósitos y extracciones de criptomonedas y moneda Fiat.

Este proyecto nace con la intención de que el usuario pueda convertirse en un pequeño inversor de manera rápida, sencilla y a bajos costos de comisión.

La tecnología, el avance cultural de distintos actores de la comunidad y el aprovechamiento de una crisis económica y social producida en Argentina, son las causas por la que los argentinos comenzaron a invertir más en criptomonedas que en pesos argentinos (ARS) y en dólares estadounidenses (USD). Esto, surge como consecuencia de una inflación 


\section{CONSTITUCIÓN Y MARCO LEGAL DE \\ UN EXCHANGE EN ARGENTINA}

mantenida desde hace años que concluye en una crisis económica, social y sanitaria que trae la pandemia del Covid-19, acompañada de una fuerte restricción en el ahorro impuesta por el gobierno.

La utilización de criptomonedas, además de ser usada como ahorro, sirve para combatir al sistema financiero tradicional. Esto es, mediante la posibilidad de realizar transferencias de criptomonedas equivalente a moneda Fiat con un mínimo de comisión.

Según una encuesta, llevada a cabo por Paxful en agosto del 2020, el 74,3\% de la población argentina considera a las criptomonedas como la mejor inversión para proteger sus fondos en tiempo de crisis. El 67,8\% de los encuestados considera que las inversiones en criptomonedas son extremadamente seguras, si se comparan con las monedas fiduciarias a nivel mundial. La misma, también reveló que un 24\% no quiere invertir en criptomonedas por falta de familiarización con la tecnología, un 12,8\% por falta de dinero y un 7,7\% por miedo a lo desconocido. Se tomó como muestra, 1113 personas en un rango de edad entre 18 y 55 años, mayoritariamente de sexo masculino.

Recuperado de https://noticias.cryptomkt.com/es/encuesta-de-paxful-argentinoscreen-que-invertir-en-criptomonedas-es-la-manera-mas-efectiva-de-ahorrar/

Con motivos de este trabajo final, realicé una encuesta similar en la provincia de Santiago del estero, en el cual surgen resultados diferentes. Cabe destacar, que la misma se realizó a 170 personas, sin distinción de sexo, con un rango de edad de 23 a 40 años. Los resultados fueron los siguientes:

- Un $68,82 \%$ invierte en USD.

- Un 38,18\% invierte en criptomonedas.

- Un $71,79 \%$ de la muestra, no invierte en criptomonedas por desconocimiento.

- Un 28,21\% no invierte en criptomonedas por falta de dinero.

- $\quad$ El 63,33 \% desconoce el significado de Exchange.

- $\quad$ El $70 \%$ adquiere sus criptoactivos en un Exchange. Mientras que el resto, lo realiza en operaciones Peer-to-Peer (P2P) o Face-to-Face (F2F). 


\section{CONSTITUCIÓN Y MARCO LEGAL DE \\ UN EXCHANGE EN ARGENTINA}

La primera encuesta nos demuestra como la mayoría de las personas confían y deciden invertir más en criptomonedas que en moneda Fiat. La duda de esta misma, radica en cuantas de estas personas tienen conocimiento en lo que están invirtiendo, o si solo lo hacen por miedo a una constante devaluación del peso. Si tenemos en cuenta la segunda encuesta, realizada en la provincia de Santiago del Estero, se pudo deducir lo contrario. La mayoría de las personas no invierte en criptomonedas, por desconocimiento y falta de información. Sin embargo, si lo hacen en moneda extranjera debido a la desconfianza que presenta el ARS. Estos resultados demuestran, una falta de educación financiera en la población analizada. Debido a que, en la actualidad el concepto de bitcoin fue escuchado en algún momento por más de una persona. Como un efecto dominó, la mayoría de la población al desconocer sobre criptomonedas, también desconoce el concepto de Exchange. Esto, representa un dato importante para este trabajo final, ya que genera la inquietud de conocer cuántas personas operarían con Cripto del Estero.

Además, el porcentaje de la población, que tiene conocimiento del concepto de Exchange, prefiere comprar los criptoactivos por dichas plataformas, y no a través de operación P2P o F2F. Desde el punto de vista del ciudadano, las operaciones P2P o F2F brindan el beneficio de menores costos frente a un Exchange.

\section{MARCO TEÓRICO}

En los últimos años, la revolución tecnológica trajo aparejada la posibilidad de poder tener relaciones financieras entre individuos sin la necesidad de un tercero. De esta manera fueron más las personas que confiaron en este ecosistema, en donde interviene no solo una decisión psíquica sino también una decisión monetaria de confianza en un sistema desconocido que se plasmó sobre conceptos como la descentralización, anonimato, algoritmo de consenso, etc.

Este ecosistema, denominado Blockchain, es el que se toma como base para esta investigación. El mismo, se define como: 


\section{CONSTITUCIÓN Y MARCO LEGAL DE \\ UN EXCHANGE EN ARGENTINA}

"Red distribuida basada en el encadenamiento de bloques vinculados entre sí, mediante hashes que permiten verificar la inalterabilidad y trazabilidad de los datos.” (Schmidt, 2020)

Otros autores, en vez de red, definen a la blockchain como "una base de datos única, descentralizada y organizada en una cadena creciente de bloques, relacionados algorítmicamente y que registran una serie de transacciones o acontecimientos digitales" (Grimaut, 2020, pág. 11).

"El sistema blockchain es a la confianza lo que internet a la información. Igual que el internet original, las cadenas de bloques tienen la capacidad de transformarlo todo." (Tapscott, 2017, pág. 6).

En definitiva, las características que se repiten son las siguientes:

- Base de datos distribuida y descentralizada.

- Inmutable.

- Anonimato entre los usuarios de la blockchain.

- Transparencia al momento de realizar las operaciones.

Es por la Blockchain que se presentan nuevas alternativas de ganancias como el Exchange, ya que permite optimizar e interactuar a través de la misma.

Cuando un individuo desea invertir en criptomonedas puede hacerlo a través de cuatro maneras distintas:

1. Minando: Esto es similar a buscar oro en una montaña, pero en una era tecnológica, es decir, a través de una computadora especializada para estas operaciones que realizan cálculos matemáticos que ayudan a la red blockchain. Por esa razón, la retribución es en criptomonedas, más la comisión que los mineros cobran por la transacción. "Los mineros a su vez generan y validan los bloques que conforman el gran libro de contabilidad que supone la red blockchain. La misión de la minería es básicamente certificar que nadie usa las monedas dos veces y que nadie pueda introducir en el mercado BTC falsos" (bit2me, 2020).

2. A través de una operación P2P o F2F: La primera implica un intercambio con otro individuo sin que tenga que intervenir un Exchange. Es decir, ambas partes tendrán sus wallet, que serán su herramienta para poder realizar la operación de compra o venta de 


\section{CONSTITUCIÓN Y MARCO LEGAL DE \\ UN EXCHANGE EN ARGENTINA}

criptomonedas. También, se acuerda la forma de operación, sin importar el lugar en donde se encuentren las partes. En cambio, en las operaciones F2F, la operación se realiza en el lugar donde las partes se encuentran físicamente, y por lo general, el pago se hace en efectivo. Esto tiene como gran ventaja el anonimato bancario.

3. Exchange: Se detallará como tema de estudio en las secciones siguientes de este trabajo final.

4. Cobro por venta de bienes o prestación de servicios (incluidos los salarios): Esto en Argentina es muy poco frecuente, se suele ver cómo, en un grupo de Facebook llamado Bitcoin Argentina, se venden productos a cambio de BTC. Pero a nivel mundial este uso es una tendencia que últimamente demuestra un gran crecimiento.

\section{Exchange}

Hablar de Exchange implica definir "una plataforma de negociaciones virtuales con criptodivisas, que permite a los clientes (traders) la realización de operaciones de compra/venta de criptomonedas y/o de intercambios de un tipo de criptomoneda por otro o por dinero Fiat y, hasta por mercancía en algunos Exchange" (cointelegraph, 2020). En este sentido, los Exchange se pueden comparar a las casas de cambio que trabajan con dinero fiduciario en el mundo real.

Podemos determinar, que un Exchange se asimila al concepto de bolsa de comercio, ya que no solo se intercambia criptomonedas sino también infinidad de activos digitales como bonos o acciones de empresas. Los inversores pueden fijar el precio a través de la oferta y demanda, por medio de órdenes de compra y venta. En otros casos, se asimila a las características de un banco como, por ejemplo, el Exchange Buenbit o Ripio porque estos deciden qué moneda ofrecer y cuáles no. Entonces, demuestran un mayor control y limitan en gran parte al usuario a seguir sus normas, políticas y términos y condiciones de dichas plataformas.

Además, los mercados de las criptomonedas reflejan una ventaja al tener movimientos 24/7/365. Es decir, 24 horas los 7 días de la semana por los 365 días del año. Esto muestra 


\section{CONSTITUCIÓN Y MARCO LEGAL DE \\ UN EXCHANGE EN ARGENTINA}

una diferencia con las bolsas de comercio, las cuales operan solamente en horarios y días hábiles.

Los Exchanges, al considerarse un tipo de entidad, con sus características pierden el potencial máximo que brindan las características de la blockchain y del mundo cripto. Ya que, al operar con estos se pierde la característica de anonimato, debido a la solicitud de datos personales, tales como, fotos, selfies, etc. También se pierde la descentralización brindada por la blockchain, para convertirse en la gran mayoría en un sistema centralizado. Esto se debe, a que el manejo de estas empresas es cerrado, imposibilitando el manejo del criptoactivo real perteneciente al usuario. Dicho de otra manera, si un usuario tiene un criptoactivo guarecido en un exchange, corre el riesgo de perderlo por posibles ataques, estafas y hackeos hacia el exchange.

La operatoria más sencilla de una plataforma de intercambio de criptomonedas es el depósito de moneda fiduciaria y/o cripto, para adquirir su viceversa. Esto es posible, ya que la plataforma presenta vinculaciones con las blockchains y protocolos de las diferentes criptomonedas ofrecidas. Una vez depositado el activo, se procede a generar órdenes de compra y/o venta, de acuerdo a la necesidad del usuario. Cuando se realiza el trade o, mejor dicho, intercalación entre las ordenes en un par dado, se procede a realizar el retiro voluntario del activo. Cuando hablamos de par dado, decimos que este, es una relación directamente proporcional del valor de un activo versus otro activo. Como, por ejemplo, el par BTC/ARS, Esto quiere decir que un BTC es equivalente a tantos ARS. Por consecuencia del trade producido por dicha operatoria, se obtiene una comisión de ganancia establecida de antemano por el exchange.

Podemos decir, que en Argentina los exchanges más conocidos son: ArgenBTC, Ripio, SatoshiTango, Qubit, Buenbit, y Bitex, entre otros. Y a nivel internacional se destacan: Binance, Coinbase, Bitstamp, Kraken, Huobi, itbit, entre otros. Los Exchanges presentan una constante expansión que se ha diversificado en función del uso y necesidad del cliente. Los Exchanges se pueden clasificar en dos grandes grupos. Por un lado, se tienen a los Exchange centralizados (CEX), y por el otro a los Exchange descentralizados (DEX). 


\section{CONSTITUCIÓN Y MARCO LEGAL DE \\ UN EXCHANGE EN ARGENTINA}

\section{Exchange Centralizado}

Desde sus inicios las criptomonedas tuvieron como característica fundamental la descentralización. No obstante, la mayoría de las negociaciones se realizan a través de medios centralizados como es el caso de la mayoría de los exchanges. Estos, son empresas o entes mediadores, quienes fijan las tasas de cambio, comisiones, políticas, reglas y términos de las mediaciones y transacciones entre los usuarios. En muchos exchanges existe una tercera persona de confianza, que intermedia entre las negociaciones realizadas por los clientes, o traders a través de la plataforma. En este sentido, los defensores de este tipo de negociación alegan que mediante la centralización se aporta liquidez, dinamismo y expansión al mercado online de criptomonedas. Entonces, el carácter descentralizador de las negociaciones se desvanece, al existir un tercero que medía entre éstas. En este caso, la persona o empresa centralizadora es quien controla las entradas y salidas en la plataforma. Además, es la que recibe una comisión por cada negociación que realicen sus clientes en sus plataformas. Entre los Exchange centralizados más populares se pueden mencionar algunos como: Binance, Bitfinex, Bittrex, Coinbase, Kraken, entre muchos otros que se pueden clasificar en esta categoría. Estas plataformas cumplen con las normas de "Conoce a tu Cliente" y "Anti lavado de Dinero" conocidas como normas KYC y AML respectivamente. Esto implica que los usuarios deben identificarse debidamente antes de poder usar los servicios que la plataforma ofrece. Por lo tanto, no son plataformas que le dan privacidad y anonimato a las negociaciones. También, cabe destacar que existen varios niveles de identificación del usuario. Mientras mayor nivel de identificación, se solicitarán mayores datos y por ende, su margen de operatoria será aún mayor.

\section{Ventajas de los exchanges centralizados}

1. Son fáciles de usar, ya que presentan interfaces de usuarios agradables para el usuario final que tiene un conocimiento básico de criptomonedas. 


\section{CONSTITUCIÓN Y MARCO LEGAL DE \\ UN EXCHANGE EN ARGENTINA}

2. Su manera de operar se actualiza constantemente, para brindar un mejor servicio y fidelidad del cliente.

3. Tienen una mayor liquidez, es decir, mayor ganancia por sus comisiones.

\section{Desventajas en los exchanges centralizados}

1. El control centralizado que se ejerce sobre los fondos.

2. No existe privacidad del usuario.

3. El riesgo de hackeo es mayor, debido a la centralización de los datos.

Los montos que se cobran por concepto de comisiones son mayores, en comparación con los DEX.

\section{Exchange Descentralizado}

En los DEX no existe el ente mediador entre los clientes. El control de las negociaciones se realiza a través de un software especializado. Este permite, que los clientes puedan realizar sus transacciones en modo P2P. Al no existir una tercera persona que intermedie entre las partes, las tasas de cambio son fijadas por los propios clientes al momento de realizar la negociación. Al tratarse de negociaciones directas entre los clientes, el sistema aporta el carácter de privacidad y anonimato entre las partes que negocian. En este sentido, muchas personas y gobiernos argumentan que este tipo de negociación, al no ser reguladas por un tercero de confianza, se preste para actividades ilícitas, como el lavado de dinero y el financiamiento de actividades que atenten contra el bien común de la sociedad. Algunos ejemplos de DEX son: Bitsquare, Changelly, Localbitcoin, EtherDelta, OpenLedger, Shapeshift y Waves. Actualmente, varios exchanges centraliados, ofrecen un servicio llamado operaciones Over the table (OTC). En estas mismas, los precios de los trades son regulados

por las partes, haciendo que el exchange no regule totalmente la operación. Ejemplos de estos, son Binance, Huobi, OkCoin, entre otros. En Argentina, la plataforma mayormente usada es LocalBitcoin y Binance. 


\section{CONSTITUCIÓN Y MARCO LEGAL DE \\ UN EXCHANGE EN ARGENTINA}

\section{Ventajas de los exchanges descentralizados}

1. La posibilidad de un control absoluto del dinero de los usuarios.

2. Al igual que el anonimato que brinda la red blockchain estos tipos de exchange brindan ese anonimato a sus usuarios.

3. Las comisiones que se pagan en estos exchanges suelen ser menores que en los centralizados.

4. Existe mayor confianza y seguridad en estas debido a que la operación se realiza con otra persona y no a través de un intermediario.

\section{Desventajas en los exchanges descentralizados}

1. No es de fácil uso para usuarios principiantes, puesto que requiere un conocimiento más profundo del sistema.

2. Sus funciones son de momento menos avanzadas que en los exchanges centralizados.

3. La libre oferta y demanda en estas exchanges pueden generar efectos colaterales nocivos.

4. No existe tanta liquidez como en las exchanges centralizadas.

\section{Características de un exchange ideal}

Para poder determinar un buen Exchange, en el cuál invertir, es necesario que los usuarios tengan en cuenta características como:

- $\quad$ Seguridad eficiente, ya que la mayoría de los Exchanges se enfrentan al problema de los hackeos en la red.

- $\quad$ Privacidad y anonimato en operaciones que involucren a las monedas

Fiat.

- Límites de compra amplios, sin necesidad de presentar mucha información personal en el KYC. En Argentina, cuando un usuario de un exchange, 


\section{CONSTITUCIÓN Y MARCO LEGAL DE \\ UN EXCHANGE EN ARGENTINA}

realiza un deposito mayor a $\$ 200000$ ARS se solicita documentación que avale y autentique los fondos del usuario.

- Tasas de cambios razonables, al no existir una tasa de cambio oficial el usuario intentará buscar las mejores tasas. La mayoría toma como precio de referencia al precio del exchange Bitstamp y a este le adiciona el porcentaje de comisión.

- Buena reputación, es decir uno en el cual el usuario confíe no solo por sus operaciones realizadas, sino también por el tiempo en la red y por la cantidad de gente que lo utilice. Se tiene mucho en cuenta, el retardo existente a la hora de realizar una extracción del activo perteneciente al usuario.

- Comisiones razonables, por lo general se tienen comisiones por los depósitos y retiros como también por los intercambios. La mayoría de los exchanges tienen comisiones variables en función de la cantidad que se comercialice.

\section{Creación de un exchange}

En Argentina, son pocas las empresas que se definen como Exchange, ya que no solo se necesita capital financiero sino también importante capital humano especializado. Entre ellos encontramos, abogados o asesores legales, contadores, administrativos, ingenieros en informática y especialistas en tecnología de la información (TI), marketing, etc. Los asesores deben conocer, las regulaciones del país, adecuándose a las políticas y procedimientos del manual de políticas de prevención ante el lavado de activos y financiamiento terrorista como también "Know your customer" (KYC). Esto significa, "el conocer al cliente a través de la identificación y verificabilidad del mismo; el término también se utiliza para referirse a las regulaciones bancarias y anti-lavado de dinero que rigen estas actividades" (Wikipedia, 2020).

Además, se necesitarán proveedores de liquidez y gestores de pago, mucha atención a la seguridad informática y los canales de soporte para brindar una mejor atención al usuario.

Por lo general, el capital inicial de un exchange ronda entre US\$1 o US\$2 millones como es el caso del exchange Ripio o sino por un monto superior $\$ 20.000$ como es el caso de SatoshiTango. 


\section{CONSTITUCIÓN Y MARCO LEGAL DE \\ UN EXCHANGE EN ARGENTINA}

Según el website de infotechnology, un exchange:

Se puede desarrollar in-house, dejándolo en manos de un equipo especializado de programadores. El beneficio de esto es que se puede fabricar el software a medida, incorporando diferentes lenguajes para diferentes criptomonedas. A la vez que se hace más sencillo agregar otras plataformas de pago (tanto de bancos como de otros exchanges) y servicios como Application Programming Interfaces (APIs).

Lo más importante es estimar los costos y el tiempo asociados con el desarrollo, el mantenimiento y la actualización constante del software en la empresa. Debido a su naturaleza compleja inherente, desarrollar un software de intercambio desde cero es bastante costoso y podría demorar entre uno y dos años (dependiendo de la experiencia de los desarrolladores) hasta que funcione correctamente.

Algunos productos ya vienen armados y son plataformas que no necesitan desarrollo. Las hay de dos clases: las abiertas y gratuitas y las privadas. En las open source, familiarizarse con el funcionamiento del script llevará tiempo y modificaciones que permiten la personalización, también demandan tiempo y dinero adicional en comparación con las

privadas. No hay garantía de que el script sea seguro y confiable. Puede ser defectuoso, susceptible a intentos de pirateo o, en el peor de los casos, incluye líneas de código maliciosas, que extraen lenta pero imperceptiblemente los fondos. La cantidad de soporte de desarrollo en un sistema de código abierto es bastante escasa, por la propia naturaleza de los desarrollos de código abierto sin propietarios. (infotechnology, 2020)

Cripto del Estero trabajará no solo con criptomonedas convencionales, sino también, con stablecoins debido a la gran demanda. El usuario, desea poder ahorrar en una moneda equivalente al USD, Yuan, etc, ya que el sistema bancario tradicional impone limitaciones para su compra.

\section{Marco Legal}

En Argentina, las criptomonedas no están reguladas al cien por ciento, y por lo general como en todos los países, esto se debe a una imposibilidad por parte del Estado. Ya que, no se 


\section{CONSTITUCIÓN Y MARCO LEGAL DE \\ UN EXCHANGE EN ARGENTINA}

puede determinar la propiedad, territorialidad y el momento en que se adquieren las criptomonedas, debido a sus características de anonimato.

Actualmente en Argentina, no existe una legislación específica acerca de los criptoactivos. Pero, podemos tener en cuenta ciertas especificaciones o regulaciones ya existentes de distintos organismos, que hablan respecto de monedas digitales y criptoactivos.

Un exchange de criptomonedas no es lo mismo que una sociedad de bolsa, estas últimas se encuentran bajo la órbita de la Comisión Nacional de Valores (CNV) y reguladas por la misma. Por ende, las criptomonedas al no contar con una regulación específica, no están reguladas por la CNV. Además, en las sociedades de bolsa, la CNV regula las sociedades tradicionales, para operar bonos y acciones. Estas se encuentran resguardadas en una Caja de Valores de Argentina, en cambio, las criptomonedas están resguardadas en la Blockchain.

Es por eso que los exchanges tienen una estructura legal de sociedad anónima (S.A.) o sociedad de responsabilidad limitada (S.R.L). Además, están inscriptas en AFIP cómo Responsables Inscriptos. Las actividades en las que se encuadran estas, son servicios de informática, y/o venta al por mayor de equipos, periféricos, accesorios y programas informáticos.

Desde un punto de vista de control financiero, la UIF (Unidad de Información Financiera) en su Resolución $N^{\circ}$ 300/14 adoptó una definición para las llamadas "monedas virtuales" considerándolas como toda representación digital de valor que puede ser objeto de comercio digital y cuyas funciones son la de constituir un medio de intercambio, y/o una unidad de cuenta, y/o una reserva de valor, pero que no tienen curso legal, ni se emiten, ni se encuentran garantizadas por ningún país o jurisdicción. En este sentido las monedas virtuales se diferencian del dinero electrónico, que es un mecanismo para transferir digitalmente monedas fiduciarias, es decir, mediante el cual se transfieren electrónicamente monedas que tienen curso legal en algún país o jurisdicción. Dicha resolución impone dos obligaciones a ciertos sujetos obligados ante la UIF con respecto a las monedas virtuales: 1) reportar información a la misma de todas las operaciones que se realicen con monedas digitales a través del reporte sistemático mensual y 2) que presten especial atención a las operaciones con 


\section{CONSTITUCIÓN Y MARCO LEGAL DE \\ UN EXCHANGE EN ARGENTINA}

criptomonedas de acuerdo al volumen y capacidad de cada uno de sus clientes dentro del marco de sus programas de conocimiento del cliente. (económica, 2020) Cabe destacar, en la resolución anteriormente detallada, que los sujetos obligados por la UIF en cuestión, son los Exchanges. Estos deben presentar los reportes hasta el quince de cada mes y dejar asentado las operaciones del mes calendario inmediato anterior. "Las sociedades dedicadas exclusivamente al intercambio de activos virtuales no se encuentran comprendidas en el art. 1 de la ley de entidades financieras, y por ende no requieren de su autorización para funcionar, lo que directamente los excluye del listado de sujetos obligados" (económica, 2020). Cabe mencionar que, hasta el momento, no se ha emitido ninguna orden dentro de la regulación de las criptomonedas en Argentina para que los exchanges que operan dentro de esta jurisdicción impongan un control sobre la cantidad de criptomonedas que pueden comprar los clientes argentinos que operan en ellos. La UIF, ante la trascendencia de las monedas virtuales en la economía mundial, se hace eco de las recomendaciones del Grupo de Acción Financiera Internacional (GAFI). Este considera, que las criptomonedas involucran una serie de riesgos para el sistema de prevención de los delitos de Lavado de Activos y de la Financiación del Terrorismo por el anonimato e imposibilidad de trazabilidad del origen de las mismas, entendiendo que las mismas pueden provenir de transacciones en países donde no existe un sistema de control y prevención. Asimismo, la AFIP dictó la resolución 4614/2019 mediante la cual establece un régimen informativo que deben cumplir los sujetos que administran, gestionan, controlan o procesan movimientos de activos a través de plataformas de gestión electrónicas o digitales, por cuenta y orden de personas humanas y jurídicas residentes en el país. es decir, los Exchanges. Este, debe informar los montos expresados en moneda de curso legal, equivalentes a las criptomonedas que el usuario posee en su plataforma. El calculo se realiza aplicando el último valor de cotización de tipo comprador, que para la criptomoneda se trate. Por ejemplo, si el usuario posee 0.01 BTC, y el tipo de cambio comprador a la fecha de cierre de mes es de 2452009 BTC/ARS, entonces el exchange debe declarar que el usuario posee $2452009 * 0.01=24520$ ARS. En este sentido, deben informar el monto total de los ingresos y egresos efectuados, así como también el tipo de ingreso (efectivo, transferencia 


\section{CONSTITUCIÓN Y MARCO LEGAL DE \\ UN EXCHANGE EN ARGENTINA}

bancaria, en moneda extranjera, moneda digital) además de especificar el saldo mensual de las cuentas en pesos argentinos, en moneda extranjera y/o en moneda digital o criptomoneda.

En relación a los impuestos de Argentina, los Exchanges deben tributar sobre: 1. Impuesto a las ganancias de personas jurídicas. 2. Impuesto al valor agregado. 3. Impuesto sobre los ingresos brutos. 3. Impuesto Bienes Personales Acciones y Participaciones. 4. Régimen de Empleadores.

Sin perder la visión general del impuesto, un Exchange es una empresa que produce ganancias o pérdidas y las mismas están inscriptas por la actividad que realizan. Por ende, estas tributan impuesto a las ganancias de personas jurídicas como tercera categoría, aplicando el criterio de imputación de lo devengado. Con la actualización de la ley 27.430 para todos aquellos ejercicios iniciados hasta el 31/12/2020, la alícuota para la misma será de 30\% mientras que para los ejercicios que comiencen al 01/01/2021 la alícuota disminuirá a un 25\%. La base imponible dependerá de qué tipo de actividad realice el mismo, pero por lo general, los exchanges cobran una comisión por las operaciones que sus usuarios realizan. Entonces, en principio, sobre esta es que se debe aplicar la alícuota general del impuesto. Es decir, la ganancia es por la diferencia entre la compra y la venta. En el caso de Cripto del estero, la venta y los préstamos, de criptomonedas, efectuados a casas de cambio están gravadas. También, lo estará la venta de stablecoin, es decir, las operaciones con monedas estables. Su gravabilidad dependerá, de si se considera dinero electrónico (moneda fiduciaria electrónica). En este caso, si es electrónico no será gravado, ya que se igualará al dinero fiduciario, el cual no está gravado. Pero si tiene respaldo en criptomonedas entonces esta actividad estará gravada.

Por otro lado, con respecto al costo computable, el art. 67 de la LIG sostiene que cuando se enajenen monedas digitales el costo a imputar será igual al valor impositivo que se les hubiere asignado en el inventario inicial correspondiente al ejercicio en que se realice la enajenación. En caso de tratarse de adquisiciones efectuadas en el ejercicio, el costo computable será el precio de compra. Y el artículo agrega que se considerará sin admitir prueba en contrario que los bienes enajenados corresponden a las adquisiciones más antiguas de su misma especie y calidad. En caso de los sujetos empresa sí estará alcanzado el resultado por 


\section{CONSTITUCIÓN Y MARCO LEGAL DE \\ UN EXCHANGE EN ARGENTINA}

tenencia. Asimismo, en el caso de personas jurídicas, los quebrantos originados en la enajenación de monedas digitales también poseen el carácter de específicos. (Zocaro, 2020, pág. 19)

La comisión que reciba un Exchange por el servicio de intermediación entre compradores y vendedores de criptomonedas estará alcanzado por IVA. Ya que, es un servicio prestado a título oneroso que no se encuentra exento, y que encuadra por estar el Exchange inscripto bajo la actividad de servicios informáticos. Respecto a ingresos brutos este es un impuesto un poco más difícil de determinar si las operaciones están alcanzadas, ya que depende de la legislación de cada provincia. Según el art. ART. $187^{\circ}$ del Código Fiscal de la Provincia de Santiago del Estero.- El ejercicio habitual y a título oneroso en jurisdicción de la Provincia de Santiago del Estero del comercio, industria, profesión, oficio, negocio, locaciones de bienes, obras o servicios, o de cualquier otra actividad a título oneroso, cualquiera sea el resultado económico obtenido y la naturaleza del sujeto que la preste incluidas las sociedades cooperativas y el lugar donde se realice (zonas portuarias, espacios ferroviarios, aeródromos y aeropuertos terminales de transporte, edificios y lugares de dominio público y privado y todo otro de similar naturaleza), estará alcanzado con un impuesto sobre los ingresos brutos en las condiciones que se determinan en los artículos siguientes. En consecuencia, la actividad de un Exchange (persona jurídica) estaría alcanzada en la medida que implique habitualidad, onerosidad y vinculación territorial con la jurisdicción. En este caso, también se debe inscribir en Dirección General de Rentas de la Provincia bajo el supuesto de servicios informáticos y tributar sobre el 3\% de los ingresos brutos. En el caso de impuesto a los débitos y créditos se tributa sobre los débitos y créditos de moneda Fiat, pertenecientes a retiros o depósitos en relación con los usuarios del exchange. "Cabe preguntarse si se considera, efectivamente, que las transferencias de monedas digitales no son movimientos de fondos, ya que en ese caso no se estaría frente al impuesto creado por la Ley 25.413/2001 (incluso, es importante recordar que el BCRA en 2014 sostuvo que las monedas digitales no tienen curso legal ni respaldo alguno)" (Zocaro, 2020, pág. 23). 


\section{CONSTITUCIÓN Y MARCO LEGAL DE \\ UN EXCHANGE EN ARGENTINA}

Cripto del Estero, al tener empleados esta obligados a su inscripción en el régimen de empleadores, para el corresponde pago de las contribuciones. Con respecto a la emisión de facturas o comprobantes que respalden las operaciones de venta de monedas digitales, o incluso los pagos por compra de bienes o servicios que se cancelan utilizando criptoactivos, se observa la siguiente situación: La RG-AFIP 1415/2003 (B.O. 13/01/2003), que establece el régimen vigente de emisión de comprobantes, no especifica nada al respecto. La documentación respaldatoria de toda venta de monedas digitales, ¿encuadra en el régimen general del artículo 1 de la RG 1415? En principio no, ya que las monedas digitales todavía no están reguladas específicamente en argentina, es por eso que, en el caso de un Exchange a la hora de facturar una compra o venta de moneda digital, sólo lo hacen por la comisión por sus servicios de intermediación, sin reflejar, a veces, el monto total operado en moneda digital y en pesos. Y los conceptos utilizados por la operatoria pueden ir desde Servicio por compra o Cargos por descarga (o carga) de saldos, hasta directamente comisión por servicios de obtención de bienes digitales por su cuenta y orden.

\section{Relación de los exchanges con el sistema financiero tradicional bancario}

El sistema financiero tradicional en la actualidad presenta inconvenientes por su obsolescencia, y porque el paso del tiempo fue permitiendo una desactualización del mismo. Don Tapscott en su libro La Revolución Blockchain menciona: "El sistema financiero funciona con una tecnología obsoleta y se regula por leyes que se remontan al siglo XIX. Abunda en contradicciones y evoluciona desigualmente, lo que lo hace a veces lento, muchas veces inseguro y, casi siempre, opaco a muchos de los que participan en él”. (Tapscott, 2017) "La tecnología del registro distribuido puede liberar muchos servicios financieros de la jaula de las viejas instituciones, así como promover la competencia y la innovación. Esto es bueno para el usuario final"'. (Tapscott, 2017)

El panorama de desactualización de los bancos, en relación a la tecnología blockchain, implica crear lazos entre los exchanges y los bancos. Esto es, no solo para una mejor administración de los fondos, sino también para generar mayores beneficios a los usuarios de 


\section{CONSTITUCIÓN Y MARCO LEGAL DE UN EXCHANGE EN ARGENTINA}

los mismos. Actualmente, la Comunicación A6823 del 31/10/2019, impide la utilización de tarjetas de crédito (emitidas por entidades locales) para la adquisición de criptoactivos en sus distintas modalidades en exchanges del exterior. Luego de esta comunicación, se generó una confusión respecto a si en Argentina se podía o no comprar criptomonedas con tarjeta de crédito en exchanges locales. La realidad es que, en el caso de los exchanges locales, las entidades financieras nunca permitieron realizar cobros a sus clientes a través de sus tarjetas de débito o crédito. Esto generaba una disyuntiva de la prohibición algo nunca sucedido.

Cabe destacar que, al menos hasta la fecha, "la compraventa de estos activos no está sujeta al cepo cambiario ni a las demás restricciones establecidas por el BCRA y la CNV sobre los denominados dólar bolsa (MEP) y contado con liquidación (CCL)" (tributum, 2020). Por tal motivo, se ha incrementado sustancialmente la operatoria con criptomonedas en los últimos meses. Cuyo principal objetivo, es la adquisición indirecta de dólares, evitando los límites establecidos en el Mercado Único y Libre de Cambios (MULC), pero a un tipo de cambio implícito más alto que el oficial y conocido popularmente como dólar crypto. Este implica la adquisición de una moneda digital con ARS, y en forma automática se vende esa misma moneda digital en USD. Para la apertura de un Exchange local, que acepta moneda FIAT de intercambio, se debe abrir una cuenta bancaria cumpliendo con los documentos como el manual de política de prevención ante el lavado de activos y financiamiento terrorista. Entre los bancos más comunes, para la apertura se encuentra: el Banco Comafi en Argentina o en el Banco industrial de Guatemala, claro que por sus facilidades en relación a los costos y documentación solicitada. Los usuarios pueden comprar activos digitales con el saldo que tienen en sus cuentas bancarias, y mover a sus cuentas bancarias lo realizado por la venta de sus activos digitales. Con lo cual, los bancos y las empresas cripto trabajan codo a codo. Entonces, en la medida en que esas relaciones existen, se necesita un marco normativo claro. Recientemente se formuló un proyecto de ley que hace referencia a las transacciones y operaciones civiles y comerciales de criptoactivos en Argentina. Se pretende expedir licencias para operar, la misma, debe ser expedida por la autoridad competente. También, se busca brindar información obligatoria, para los usuarios en un esquema similar al que rige para la 


\section{CONSTITUCIÓN Y MARCO LEGAL DE \\ UN EXCHANGE EN ARGENTINA}

operación de bancos y otras entidades financieras tradicionales. Además, el proyecto de ley presenta la posibilidad de generar sanciones a cargo de organismos oficiales. Según Ignacio Torres, diputado opositor de Juntos por el cambio, autor del proyecto, esto aplicará a las firmas que estén radicadas o tengan actividad en el país. Por último, la norma designa al Banco Central, la UIF o la CNV como las entidades que podrán sancionar o revocar las licencias operatorias si hubiera incumplimientos. Insistió en que su iniciativa no implica un costo fiscal. Conjuntamente, un diputado del frente para todos presenta un proyecto similar que apunta a "cuatro principios: protección al consumidor; la prevención del fraude y otras actividades delictivas, promoción de la competencia privada y la innovación tecnológica”. (nación, 2020)

\section{CONCLUSIÓN}

El presente trabajo apunto a dar claridad a la creación de un exchange en Argentina. Despejando dudas respecto a contenidos básicos y necesarios como blockchain, exchange y su marco legal. Diferenciando los impuestos que gravan al exchange, y que son muy distintos a los impuestos del ciudadano con tenencia de criptomonedas. En conclusión, queda demostrado que una falta de regulación específica y un marco normativo no protege ni a los usuarios, ni a los exchanges. Como tampoco genera una claridad respecto de la prevención de lavado de activo, ni financiamiento terrorista. Al no estar regulado, tampoco genera confianza en el sistema financiero tradicional para poder acceder a distintos tipos de préstamos. Se necesita una normativa clara que genere un beneficio para todos y que, en el caso de aprobarse el proyecto de ley, los criptoactivos puedan utilizarse de manera regulada como medio de pago, ahorro e inversión. Logrando de esta manera, favorecer a un esquema de desarrollo, con mayores inversiones y generación de empleos en el país. Mientras Argentina sea un país con inflación alta, sus ciudadanos tengan desconfianza en el sistema, la pobreza, el desempleo, y las barreras impuestas por el gobierno, sigan aumentando, siempre habrá un vacío en donde las criptomonedas y la tecnología blockchain puedan llenar. Porque sus características de anonimato, descentralización y bajos costos serán las más importantes a la hora de definir en qué invertir. Ya que la libertad personal y financiera serán valoradas en un contexto como el 


\section{CONSTITUCIÓN Y MARCO LEGAL DE \\ UN EXCHANGE EN ARGENTINA}

mencionado. El proyecto de la creación de Cripto del Estero es viable siempre que se cumpla con la normativa, y siempre que el ciudadano argentino apueste ya sea por educación financiera o como salida de inversión en criptomonedas.

\section{BIBLIOGRAFÍA}

bit2me. (2020). bit2me. Obtenido de https://academy.bit2me.com/que-es-minarbitcoins /

cointelegraph. (2020). cointelegraph. Obtenido de

https://es.cointelegraph.com/explained/what-is-an-exchange

cryptomkt. (s.f.). Obtenido de https://noticias.cryptomkt.com/es/encuesta-de-paxful-

argentinos-creen-que-invertir-en-criptomonedas-es-la-manera-mas-efectiva-de-ahorrar/

economica, C. d. (2020). CIPCSE. Obtenido de

http://www.cipce.org.ar/articulo/criptomonedas-argentina-proposito-del-comunicado-uif

Grimaut, A. (2020). Clase 1 - PPT -. Cordoba: Contenido propio de la Diplomatura en tecnologias Blockchain para los negocios y relaciones jurídicas.

infotechnology. (2020). infotechnology. Obtenido de

https://www.infotechnology.com/negocios/Paso-a-paso-como-crear-tu-propio-negocio-

basado-en-Bitcoin-y-criptomonedas-20171205-0002.html

nacion, L. (2020). La Nacion. Obtenido de

https://www.lanacion.com.ar/economia/ley-criptomonedas-que-dice-proyecto-se-debatira$\operatorname{nid} 2506617$

Schmidt, W. (2020). Introducción a Blockchain - Conceptos. Cordoba: Contenido propio de la Diplomatura en tecnologias Blockchain para los negocios y relaciones jurídicas.

Tapscott, D. (2017). La revolución Blockchain. DEUSTO.

tributum. (2020). tributum. Obtenido de https://tributum.news/el-marco-regulatorio-

de-las-criptomonedas-en-argentina-comparativa-con-otros-paises /

Wikipedia. (2020). Wikipedia. Obtenido de

https://es.wikipedia.org/wiki/Conozca_a_su_cliente 


\section{CONSTITUCIÓN Y MARCO LEGAL DE UN EXCHANGE EN ARGENTINA}

Zocaro, M. (2020). economicas. Obtenido de http://www.economicas.uba.ar/wpcontent/uploads/2020/07/El-marco-regulatorio-de-las-criptomonedas-en-Argentina.pdf 